\title{
A composição da avifauna do campus da Universidade Estadual de Londrina, norte do Paraná, Brasil
}

\author{
Edson V. Lopes \& Luiz dos Anjos
}

Departamento de Biologia Animal e Vegetal, Universidade Estadual de Londrina. Caixa Postal 6001, 86051-970, Londrina, Paraná, Brasil. E-mail: papaformiga@yahoo.com.br; Ilanjos@sercomtel.com.br

\begin{abstract}
The avifaunal composition of Universidade Estadual de Londrina, northern Paraná, Brazil. This study analyzed the spatial distribution of the Universidade Estadual de Londrina's (UEL) avifaunal in several habitats (forest, scattered trees, buildings and surroundings, shrubs, aquatic and grassland). Besides that, a comparative analysis about the richness and composition of species and the guild structure of forest birds was carried out between the bird community witch was recorded in a forest remnant of Universidade Estadual de Londrina campus and the bird communities of one forest remnant in a country area, two forest remnants in an urban area and a continuous forest area. Field data were derived by transect count from January 2001 to December 2002 in 48 samples. A total of 174 species were recorded, being the forest and scattered trees habitats the highest species richness, while the aquatic and the grassland habitats presented the lowest species numbers. The species richness recorded at the campus forest remnant was similar to that recorded in a continuous forest area and a country area and higher than the one recorded in a urban area. The Sørensen index showed similar and relatively high values between the campus and the four other areas. On another hand, the guild structure of forest birds of the campus showed greater similarity to that an urban area and a country area (Spearman rank correlation $\alpha=0,5$ ). At the campus and also in a urban area, a diminution of the large frugivorous and creeper species occurred and an increase of forest edge species related to a continuous forest area and a country area.
\end{abstract}

KEY WORDS. Forest remnant; guild structure; spatial distribution.

RESUMO. Este estudo analisou a distribuição espacial da avifauna do campus da Universidade Estadual de Londrina em diferentes habitats (florestal, agrupamento de árvores, edificações e adjacências, capoeira, aquático e campo aberto). Também foi desenvolvida uma análise comparativa em relação à riqueza e composição de espécies e à estrutura de guildas entre a comunidade de aves que ocorre em um remanescente florestal que existe no campus com as de um remanescente em área rural, dois remanescentes em área urbana e a de uma área contínua de floresta. Os dados foram coletados em transecções fixas entre janeiro de 2001 e dezembro de 2002 em 48 amostragens. Um total de 174 espécies foi registrado, com os habitats florestal e agrupamento de árvores apresentando maior número de espécies, enquanto os habitats aquático e de campo aberto apresentaram o menor número. A riqueza de espécies registrada no remanescente florestal do campus foi semelhante ao registrado na área contínua e na área rural e superior ao registrado nas duas áreas urbanas. O índice de Sørensen apresentou valores semelhantes e relativamente altos entre o campus e as outras quatro áreas. Por outro lado a estrutura de guildas da comunidade de aves florestais do campus foi mais semelhante o de uma das áreas urbanas e da área rural. Tanto no campus como nas áreas urbanas, houve menor número de espécies de grandes frugívoros e escaladores e um aumento na representatividade de aves de borda de floresta em relação a área contínua de floresta e a área rural.

PALAVRAS CHAVE. Distribuição espacial; estrutura de guildas; remanescente florestal.

Com a ocupação humana, a floresta contínua do norte do Paraná cedeu lugar a uma paisagem em mosaico, composta por fragmentos relativamente isolados entre si. Na região de Londrina, no norte do estado, os remanescentes florestais, que dificilmente ultrapassam 100 ha de área, somam apenas $2-4 \%$ da cobertura original (IPARDEs 1993). Uma das maiores e mais bem preservadas áreas de floresta da região é o Parque Estadual Mata dos Godoy, com 656 ha de floresta estacional semidecídua. Representantes arbóreos típicos desta formação são: Euterpe edulis Mart. (Arecaceae), Ficus glabra Vell. (Moraceae), e Aspidos- 
perma polyneuron Müll. Arg. (Apocynaceae). Este parque vem sendo utilizado, como área referencia da diversidade de aves original da região (ANjos et al. 1997, ANJos 2001, 2002a, b).

Os fatores mais enfocados nos estudos sobre o efeito da fragmentação do habitat florestal são o tamanho do remanescente e o seu grau de isolamento ou conectividade; o tempo pós fragmentação é menos freqüentemente abordado (GALli et al. 1976, BierregaArd \& Stouffer 1997, Anjos \& Boçon 1999, ANJos 2001, Davies et al. 2000). Os estudos sobre os efeitos derivados do ambiente que compõe o entorno da área do remanescente, ou seja, a matriz na qual o remanescente está inserido tem recebido cada vez mais atenção (STOUfFER \& BierREgaARD 1995, Warburton 1997, Gascon et al. 1999, Antongiovanni \& Metzger 2005, Uezu et al. 2005).

$\mathrm{O}$ crescimento dos centros urbanos insere mais um componente de pressão no processo de fragmentação do habitat para a flora e fauna residual. Remanescentes florestais, inseridos em uma matriz totalmente urbana, densamente povoada, possivelmente, são mais perturbados do que aqueles mais afastados de aglomerações humanas e/ou inseridos em uma matriz diversificada em termos de vegetação. Fundos de vale, praças e parques são exemplos de áreas verdes inseridas na malha urbana das cidades que podem abrigar certa diversidade (ARGEL DE Oliveira 1990, 1995, Matarazzo-Neuberger 1995, Westcott et al. 2002). Entretanto, a maioria das aves que ocorrem nestes locais representam nova colonização, pois geralmente são locais reflorestados, com forte interferência humana na composição e estrutura da vegetação. Enquanto que em um remanescente florestal, mesmo inserido em uma matriz urbana, podemos encontrar uma maior proporção de representantes da avifauna que ocorria antes da remoção da floresta (ANJOs \& LAROCA 1989, KRÜGEL, \& Anjos 2000). Áreas de campus de Universidades geralmente abrigam grande número de espécies (Matarazzo-Neuberger 1990, Höfling \& Camargo 1999, Monteiro \& Brandão 1995).

No presente estudo a composição da avifauna do campus da UEL é analisada em relação à distribuição espacial em diferentes habitats. Também são desenvolvidas análises comparativas entre a comunidade de aves que ocorre em um remanescente florestal de 10 ha que existe no campus da UEL com as de 1) um remanescente de tamanho semelhante, em área rural, 2) dois remanescentes em área urbana, um de tamanho semelhante e um maior e 3) a do Parque Estadual Mata dos Godoy. Estas comparações foram desenvolvidas em relação à riqueza e composição de espécies e à estrutura de guildas das comunidades.

\section{MATERIAL E MÉTODOS}

\section{Área de estudo}

A área ocupada pelo campus da UEL em Londrina, amostrada neste estudo possui 222 ha (Fig. 1). Em princípio isolado da malha urbana, hoje faz divisa ao norte, ao leste e a oeste com a periferia da cidade, e ao sul com a zona rural do município.

Seis ambientes foram considerados no campus da UEL, para a realização das amostragens: 1) florestal (FL), composto

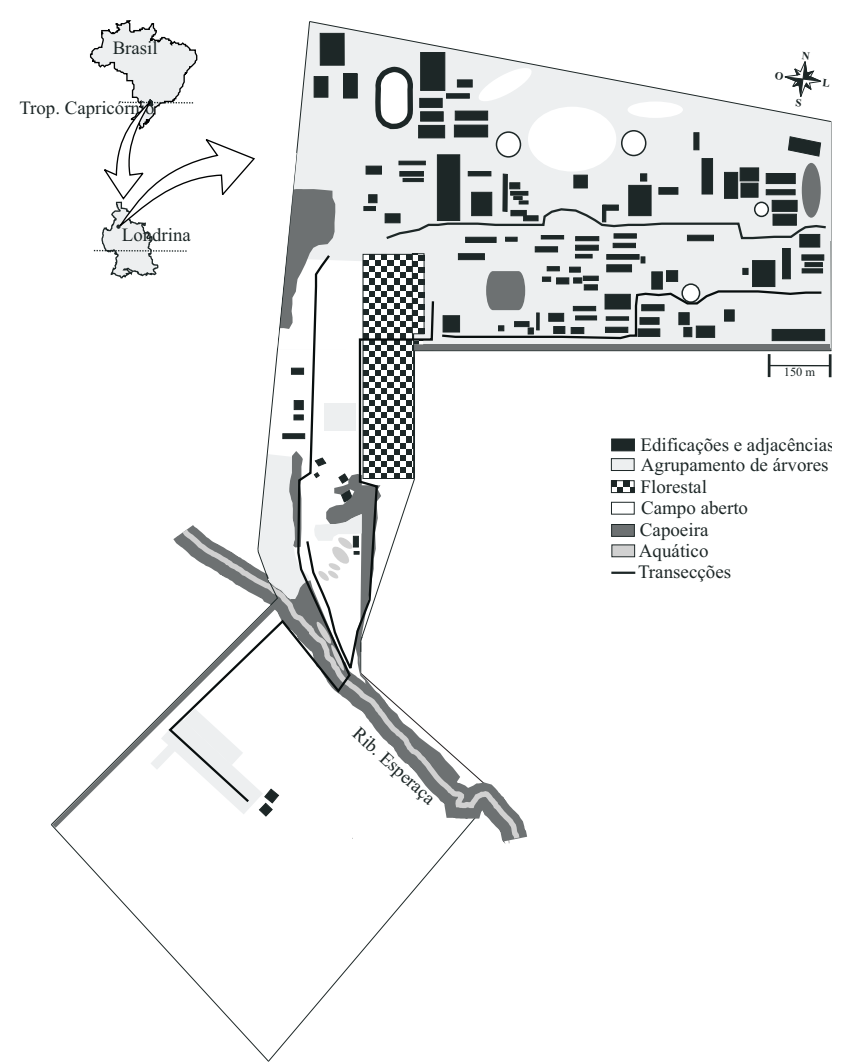

Figura 1. Distribuição dos diferentes habitats no campus da Universidade estadual de Londrina.

por remanescente de floresta nativa com área de 10 ha, com vegetação em estádio secundário tardio de sucessão; 2) edificações e adjacências (EA), representado por prédios, vias pavimentadas, estacionamentos e seu entorno mais próximo (até $30 \mathrm{~m})$; 3) agrupamento de árvores (AA), pomares e árvores esparsas (3-10 m) em gramado com decoração paisagística heterogênea; 4) capoeira (CP); 5) aquático (AQ), composto pelo ribeirão Esperança, o qual corta a região sul do campus, e sete lagos artificiais usados em piscicultura e 6) campo aberto (CA), composto por gramados sem árvores, área de pasto e de plantio de culturas anuais como milho e soja.

\section{Observações em campo}

Para a obtenção dos dados em campo foi utilizado o método da transecção (Biввy et al. 1992). Este método consiste em percorrer uma transecção previamente estabelecida, em uma velocidade média constante. Foram registradas todas as espécies, independentemente da forma de registro (visual e/ou auditivo) e da distância da ave em relação ao observador. Para que todos os habitats fossem amostrados foram determinadas quatro transecções com comprimento variando entre $1.000 \mathrm{e}$ $1.900 \mathrm{~m}$. Cada espécie foi registrada uma única vez em cada dia de amostragem, com isto, foi obtida a freqüência de ocorrência 
de cada espécie, que consiste no número de registros da espécie dividido pelo total de amostragens realizadas (48 amostragens).

As amostragens iniciavam ao alvorecer e encerravam cerca de três horas após. Foram realizados quatro dias consecutivos de amostragem; um dia para cada transecção a cada, aproximadamente, 60 dias, entre janeiro de 2001 e dezembro de 2002, totalizando 48 amostragens ao longo dos dois anos, sendo 12 amostragens em cada transecção. Também foram incorporadas, na listagem final, espécies de aves registradas apenas fora das amostragens. Para estas espécies não foi calculada a freqüência de ocorrência.

\section{Procedimento de análise}

$O$ teste $\mathrm{G}$ foi utilizado para comparar a riqueza de espécies de cada habitat considerado e a proporção de espécies exclusivas de cada habitat. Para esta análise foram consideradas todas as espécies registradas na UEL, incluindo as registradas fora do período amostral.

A comunidade de aves registrada no remanescente florestal do campus da Universidade Estadual de Londrina (UEL, 10 ha) foi comparada àquelas registradas nas áreas contínua de floresta (PG, 656 ha, 70 ha amostrados) e remanescente em área rural (FC, 11 ha) do estudo de Anjos (2001) e nas áreas urbanas (M4: 26,7 ha e M5: 7,6 ha respectivamente) do estudo de KRÜGUEL $\&$ Anjos (2000). As cinco áreas estão inseridas nos domínios da floresta estacional semidecídua, a UEL, PG e FC, no município de Londrina e M4 e M5 em Máringa, distante 90 km de Londrina. O Parque Estadual Mata dos Godoy (PG) foi utilizado, nas comparações, como área referencial da avifauna original da região. Para estas comparações foram utilizados: o índice de similaridade de Sørensen para a composição de espécies, o teste G para a riqueza de espécies e o coeficiente de correlação por postos de Spearman para a estrutura de guildas das comunidades. As espécies foram agrupadas em guildas conforme observações em campo e segundo Willis (1979); foram consideradas quinze guildas para a análise.

$\mathrm{Na}$ análise das aves florestais, foram excluídas: espécies consideradas migrantes, segundo sua ocorrência ao longo do ano е Sтотz et al. (1996), aves noturnas (Strigidae, Nyctibidae e Caprimulgidae), beija-flores (Throchilidae) e forrageadores aéreos como andorinhas (Hirundinidae) e andorinhões (Apodidae). Em função de peculiaridades de cada grupo, estes podem ter sido representados de forma distinta em cada estudo aqui considerado. Três outras espécies: Colaptes campestris (Vieillot, 1818), Machetornis rixosa (Vieillot, 1819) e Ammodramus humeralis (Bosc, 1792), registradas em M4 ou M5 foram retiradas das analises por serem espécies campestres, não relacionadas ao habitat florestal. Estas espécies também foram registradas na UEL e provavelmente ocorrem em habitats abertos no entorno de PG e FC.

\section{RESULTADOS}

Um total de 174 espécies foi registrado no campus da UEL (Apêndice I): 154 espécies foram registradas nas amostra- gens e 20 foram registradas fora do período amostral. A riqueza total de espécies de cada habitat foi razoavelmente obtida, como mostra a figura 2. Houve diferença no número total de espécies nos habitas estudados ( $\mathrm{G}=137,1 ; \mathrm{gl}=5, \mathrm{p}<0,001)$, com o habitat florestal (109 espécies) apresentando maior número de espécies que os habitats aquático (27 espécies) e de campo aberto (27 espécies) (Tab. I). A floresta apresentou também o maior número de espécies exclusivas (39 espécies), embora a maior proporção de espécies exclusivas tenha ocorrido no habitat aquático (56\%).

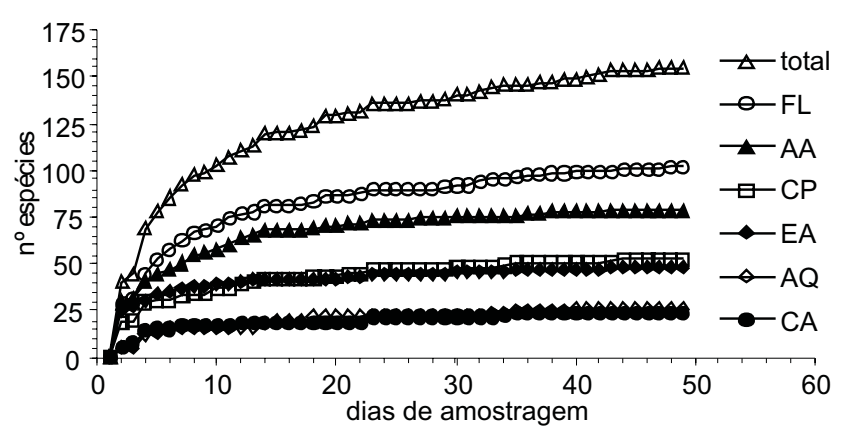

Figura 2. Curva do coletor para a riqueza total de espécies registrada na Universidade Estadual de Londrina, durante o período amostral individualmente e para os seis habitats. (FL) Florestal, (EA) edificações e adjacências, (AA) agrupamento de árvores, (CP) capoeira, (AQ) aquático, (CA) campo aberto.

Tabela I. Número total de espécies e de espécies exclusivas registrado em cada habitat amostrado na Universidade Estadual de Londrina. (FL) Florestal, (EA) edificações e adjacências, (AA) agrupamento de árvores, (CP) capoeira, (AQ) aquático, (CA) campo aberto.

\begin{tabular}{ccc}
\hline Habitat & Número de espécies & Espécies exclusivas \\
\hline FL & 109 & 39 \\
EA & 56 & 0 \\
AA & 92 & 4 \\
CP & 56 & 5 \\
AQ & 27 & 15 \\
CA & 27 & 7 \\
\hline
\end{tabular}

A riqueza de espécies na UEL e nos outros quatro locais considerados foi diferente entre as cinco áreas; UEL: 90, PG: 113, FC: 93, M4: 66 e M5: $49(\mathrm{G}=31,5 ; \mathrm{gl}=4, \mathrm{p}<0,001)$. Excluindo-se as áreas M4 e M5 da analise a diferença entre o número de espécies é não significativa $(\mathrm{G}=3,1 ; \mathrm{gl}=2 ; \mathrm{p}>0,05)$. O índice de similaridade de Sørensen aplicado entre a UEL e as 4 áreas apresentou valores semelhantes e relativamente altos: 0,74 com M4, 0,7 com FC, 0,65 com M5 e 0,64 com PG. 
A estrutura de guildas da comunidade de aves florestais da UEL foi mais semelhante à das áreas M4 $\left(\mathrm{r}_{\mathrm{s}}=0,92 ; \mathrm{p}<0,01\right)$ e FC $\left(r_{s}=0,79 ; p<0,01\right)$. Em relação a PG $\left(r_{s}=0,58 ; p<0,05\right)$ e a M5 $\left(r_{s}=0,58 ; p<0,05\right)$ a estrutura da comunidade foi marginalmente semelhante (Tab. II).

Tabela II. Número de espécies de aves florestais em cada guilda nas cinco áreas analisadas. (IFSC) Insetívoro de folha de sub-copa, (CAD) carnívoro aéreo diurno, (ESC) escalador, (FGR) frugívoro grande, (FPQ) frugívoro pequeno, (IAD) insetívoro aéreo diurno, (IBO) insetívoro de borda, ICO: insetívoro de copa, (IRB) insetívoro de reboleira, (ISC) insetívoro de sub-copa, (OBO) onívoro de borda, (OCO) onívoro de copa, (OSC) onívoro de sub-copa, (OSO) onívoro de solo, (GRA) granívoro. (UEL) Universidade Estadual de Londrina, (PG) área contínua de floresta, (FC) remanescente florestal em área rural, (M4 e M5) remanescentes florestal em área urbana.

\begin{tabular}{lrrrrr}
\hline \multirow{2}{*}{ Guildas } & \multicolumn{5}{c}{ Áreas } \\
\cline { 2 - 6 } & UEL & PG & FC & M4 & M5 \\
\hline IFSC & 4 & 7 & 4 & 3 & 2 \\
CAD & 2 & 3 & 3 & 2 & 2 \\
ESC & 9 & 14 & 14 & 5 & 2 \\
FGR & 10 & 18 & 13 & 4 & 1 \\
FPQ & 3 & 4 & 2 & 1 & 1 \\
IAD & 1 & 0 & 0 & 0 & 0 \\
IBO & 13 & 6 & 9 & 12 & 9 \\
ICO & 1 & 1 & 1 & 1 & 1 \\
IRB & 6 & 12 & 10 & 3 & 2 \\
ISC & 5 & 10 & 4 & 4 & 3 \\
OBO & 12 & 2 & 4 & 10 & 10 \\
OCO & 9 & 9 & 10 & 8 & 7 \\
OSC & 8 & 17 & 12 & 8 & 6 \\
OSO & 5 & 10 & 7 & 3 & 1 \\
GRA & 2 & 0 & 0 & 2 & 2 \\
\hline
\end{tabular}

\section{DISCUSSÃO}

A área do campus da UEL possui uma avifauna relativamente rica, se comparada ao registrado em outros (HöfLING \& Camargo 1999 [143 espécies], Monteiro \& Brandão 1995 [96 espécie]). De forma geral, a variedade de habitats que ocorre no campus da UEL parece contribuir para a relativamente alta riqueza de espécies do local, especialmente considerando o número de espécies que ocorreram em apenas um habitat (70 no total).

Mesmo representando menos de 5\% da área do campus, o habitat florestal se mostrou o mais rico em espécies, o que seria o esperado, pois é o mais complexo dos habitats estudados. A alta riqueza de espécies registrada no habitat formado por árvores esparsas era esperada em função da grande área relativa que possui e vegetação heterogênea. Além disso, este habitat compõe a matriz onde outros habitats estão inseridos. Aves que apresentam preferência por outros habitats acabam transitando pelo habitat matriz para alcançar as áreas de outros habitats, distribuídos em manchas.

O Campo aberto também possui área relativamente grande e funciona como matriz de outros habitats, contudo a falta de vegetação dificulta a colonização deste habitat, sendo responsável, ao menos em parte, pelo baixo número de espécies registradas. O baixo número de espécies do habitat aquático pode ser em função de uma maior necessidade de especialização das espécies que exploram este habitat. Além deste habitat apresentar a menor área relativa neste estudo.

O número de espécies florestais registradas na UEL está acima do encontrado nos remanescentes de Maringá e se aproxima do registrado na área referencial (PG) e no remanescente próximo desta (FC). O PG é uma área bem maior; a área FC está localizada próxima de PG e inserida em uma matriz essencialmente agropecuária, aumentando a conectividade. Os remanescentes de Maringá apresentam grau maior de isolamento e estão inseridos em uma matriz totalmente urbana, densamente povoada. Já a UEL está inserida em uma matriz com componentes urbanos e rurais e apresenta certa conectividade com outros remanescentes pequenos através da mata ripária do ribeirão que corta a área do campus.

Seria esperado que a UEL fosse mais semelhante a PG e FC do que a M4 e M5, em termos de composição especifica, pois está a cerca de $15 \mathrm{~km}$ de PG e FC e a $90 \mathrm{~km}$ de M4 e M5. Entretanto, a UEL mostrou similaridade parecida com as 4 áreas. A composição específica, pode ter sido mais influenciada pelo histórico de ocupação do local e do entorno. Neste sentido, a UEL ocupa uma posição intermediária, pois recebeu mais interferência humana do que PG e FC, mas não tanto quanto M4 e M5. Contudo, esta posição intermediária da UEL deve ser analisada com mais cautela, pois a distância entre Londrina e Maringá poderia estar influenciando a composição das espécies de cada local, em outra escala, mesmo sendo a floresta estacional semidecídua a vegetação de ambos os locais.

Variações na estrutura de guildas podem ser reflexo da extinção local de espécies e da colonização da área por outras espécies. Neste estudo foi detectado que 51 espécies do PG não foram registradas no remanescente florestal do campus da UEL. É esperado que estas espécies tenham ocorrido no passado na área do campus, mas foram extintas em função do desmatamento. Algumas espécies do PG (62 espécies), especialmente aquelas tolerantes ou associadas à borda de floresta, conseguiram se adaptar à vegetação florestal remanescente do campus. Outras 28 espécies, registradas no habitat florestal remanescente na UEL, mas não no PG, podem representar nova colonização. Um bom exemplo é o de Zenaida auriculata (Des Murs, 1847) que, em condições naturais, ocorre em áreas abertas como campos, cerrados e caatinga (MurTon et al. 1974, AguirRe 1976). Esta espécie coloniza áreas de cultivo de grãos e 
até ambientes urbanos com grande sucesso. Embora haja carência de comprovação, $Z$. auriculata, que era incomum a até algumas décadas na região de Londrina, pode ser, atualmente, a espécie mais abundante na zona rural do município, e mesmo na área urbana, onde milhares de indivíduos se reúnem para pernoitar. Este padrão de congregação em grandes bandos para algumas atividades como reprodução, alimentação e dormida, faz parte da ecologia da espécie e tem sido registrado em diversos locais dentro da sua área de distribuição, onde antes não ocorria, especialmente onde a floresta cedeu lugar a culturas anuais e cidades (Bucher 1982, RANVAUd et al. 2001).

Com a alteração da paisagem que ocorreu em função da remoção da floresta, há também exemplo de expansão da distribuição geográfica de algumas espécies. Este é o caso de Griseotyrannus aurantioatrocristatus (d'Orbigny \& Lafresnaye, 1837), que é o primeiro registro para o estado do Paraná. Alguns indivíduos desta espécie foram observados durante primavera e verão (setembro a janeiro). A distribuição conhecida desta espécie abrange estados do norte do Brasil, e outros países, desde a Venezuela, até a Argentina e Uruguai. Também ocorre no Rio Grande do Sul e no oeste e norte de São Paulo, mas não havia ainda sido registrada em território paranaense (Ridgely \& Tudor 1994, Scherer-Neto \& Straube 1995, Sick 1997, Willis \& ONIKI 2003). Em função de esta espécie ocorrer em áreas abertas e, aparentemente, ser beneficiada pelo desmatamento, o registro na UEL sugere uma colonização recente.

As aves de solo, principalmente as de maior porte como Cracidae e Tinamidae, e as que apresentam maior especialização, como insetívoros de reboleira e de sub-copa são muito sensíveis quanto a perturbações em seus habitats, muitas vezes sendo as primeiras a serem extintas em processos de fragmentação florestal (Willis 1979, BierregaArd 1990, Aleixo \& Vielliard 1995, BierregaArd \& Stouffer 1997, Anjos 2001, 2004). Tais processos favorecem espécies que habitam a borda da floresta e estas espécies podem aumentar em densidade.

A guilda das aves frugívoras de grande porte e onívoras de solo, representada principalmente por Tinamidae, Cracidae, Pscitacidae e Ramphastidae diminuiu na UEL e nas áreas de Maringá. Contudo, o número de espécies de Pscitacidae registradas na UEL foi próximo ao de PG e FC, entre elas Aratinga auricapillus (Kuhl, 1820), considerada espécie vulnerável (ColLAR et al. 1992, BirdLife InTERnATional 2000). Entretanto, estas espécies ocorreram, na UEL, com freqüência muito baixa, o que sugere que estejam apenas de passagem pelo campus da UEL. Situação semelhante ocorre na guilda dos escaladores. Neste caso, ocorreu grande diminuição, nas áreas de Maringá e na UEL, de espécies da família Dendrocolaptidae. Esta família teve apenas uma espécie (Dendrocolaptes platyrostris Spix, 1825) com um único registro na UEL e nenhuma espécie foi registrada nas áreas de Maringá, enquanto Anjos (2001) menciona cinco espécies para o PG e para FC. Aparentemente, Esta família é muito sensível à fragmentação florestal (WiLlis 1979, BiERREGAARD \& Stouffer 1997, Soares \& Anjos 1999, Aleixo 1999). Portanto é coerente que estejam ausentes ou pouco representadas em um local alterado como a área do campus ou os remanescentes de Maringá.

Aves mais generalistas quanto aos hábitos alimentares, como onívoros de borda aumentaram acentuadamente sua abundância na UEL e, principalmente em M4 e M5, em relação à PG e FC. Outras espécies podem ter sido favorecidas pelo homem, na medida em que este lhes fornece recursos, não necessitado exclusivamente da área de floresta para obtê-los, visitando-a mais esporadicamente.

Embora um remanescente florestal com pressão antrópica moderada e em contato com outros habitats, como é o do campus da UEL, não seja suficiente para manter uma comunidade de aves com estrutura semelhante ao que ocorre em uma área de floresta contínua, aparentemente, este remanescente consegue manter uma comunidade com estrutura semelhante ao que ocorre em um remanescente de mesmo tamanho, localizado próximo a uma área de floresta contínua, ou de uma área de tamanho maior, que esteja mais isolada e com forte pressão antrópica como M4.

\section{AGRADECIMENTOS}

Os dois autores são bolsistas CNPq, o primeiro de mestrado e o segundo de produtividade em pesquisa. Apoio financeiro foi concedido pelo CNPq por meio do Programa Mata Atlântica (Assessoria de Cooperação Internacional, Processo 690146/019). O Laboratório de Bioacústica da Universidade Estadual de Londrina disponibilizou seu acervo de gravações de vozes de aves o que auxiliou na identificação de algumas espécies. Fabíola Polleto e Patrícia Serafini auxiliaram nas amostragens.

\section{REFERÊNCIAS BIBLIOGRÁFICAS}

Aguirre, A.C. 1976. Distribuição, costumes e extermínio da "avoante" do nordeste, Zenaida auriculata Noronha

Chubb. Rio de Janeiro, Academia Brasileira de Ciências, 35p. Aleixo, A. 1999. Effects of selective logging on a bird community in the Brazilian Atlantic Forest. The Condor, Lawrance, 101: 537-548.

Aleixo, A. \& J.M.E. Vielliard. 1995. Composição e dinâmica da avifauna da mata de Santa Genebra, Campinas, São Paulo, Brasil. Revista Brasileira de Zoologia, Curitiba, 12 (3): 493 511.

Anjos, L. Dos. 2001. Bird communities in five Atlantic forest fragments in southern Brazil. Ornitologia Neotropical, Montreal, 12 (1): 11-27.

Anjos, L. Dos. 2002a. Forest Bird communities in the Tibagi river hydrographic basin, southern Brazil. Ecotropica, Bonn, 8 (1): 67-79.

Anjos, L. Dos. 2002b. A avifauna da bacia do rio Tibagi, p. 271290. In: M.E. Medri; E. Bianchini; J.A. Pimenta \& O.A. Shibata (Eds). A bacia do rio Tibagi. Londrina, Edição dos editores, 595p.

Revista Brasileira de Zoologia 23 (1): 145-156, março 2006 
Anjos, L. Dos. 2004. Species richness and relative abundance of birds in natural and anthropogenic fragments of Brazilian Atlantic forest. Anais da Academia Brasileira de Ciências, Rio de Janeiro, 76 (2): 429-434.

Anjos, L. Dos \& R. Boçon. 1999. Bird communities in natural Forest patches in southern Brazil. Wilson Bulletin, Ann Arbor, 111 (3): 397-414.

Anjos, L. Dos \& S. LARoca. 1989. Abundância relativa e diversidade específica em duas comunidades urbanas de aves de Curitiba (sul do Brasil). Arquivos de Biologia e Tecnologia, Curitiba, 32 (4): 637-643.

Anjos, L. dos; K.-L. Schuchmann \& R. Bernedt. 1997. Avifaunal composition, species richness, and status in the Tibagi river basin, Paraná State, southern Brazil. Ornitologia Neotropical, Montreal, 8 (2): 145-173.

Bibby, C.J.; N.D. Burgess \& D.A. Hill. 1992. Bird Census Techniques. San Diego, Academic Press, 257p.

Antogiovanni, M. \& J.P. Metzger. 2005. Influence of matriz habitats on the occurrence of insectivorous birds species in Amazônia Forest fragments. Biological Conservation, Kidlington, 122 (3): 441-451.

Argel de Oliveira, M.M. 1990. Arborização e avifauna urbana em cidades do interior paulista. Boletim CEO, São Paulo, 7 (1): $10-15$

Argel de Oliveira, M.M. 1995. Aves e vegetação em um bairro residencial da cidade de São Paulo (São Paulo, Brasil). Revista Brasileira de Zoologia, Curitiba, 12 (1): 81-92.

Bierregaard, R.O. 1990. Avian communities in the understory of Amazonian forest fragments p 333-343. In: KEAST, A. (Ed.). Biogeography and ecology of forest birds communities. The Hague, SPB Academic Publishing, 410p.

BierregaArd JR., R.O. \& P.C. Stouffer. 1997. Understory birds and dynamic habitat mosaics in Amazonian rainforest, p. 138155. In: W.F. Laurance \& R.O BierregaARd JR. (Eds). Tropical forest remnants: ecology, management, and consevation of fragmented communities. Chicago, The University of Chicago Press, XV+616p.

Birdife International. 2000. Threatened birds of the world. Cambridge, Lynx Edition and BirdLife International, $\mathrm{XII}+851 \mathrm{p}$.

Bucher, E.H. 1982. Colonial breending of the Eared Dove (Zenaida auriculata) in Oorth-eastern Brazil. Biotropica, Lawrance, 14: 225-261.

Collar, N.J.; L.P. Gonzaga; N. Krabbe; A. Madroño Nieto; L.G. Naranjo; T.A. Parker III \& D.C. Wege. 1992. Threatened birds of the Americas. Cambridge, Page Bros (Norwick), 1150p.

Davies, K.F.; C.R. Margules \& K.F. Lawrence. 2000. Which traits of species predict population declines in experimental Forest fragments?. Ecology, Washington, 81: 1450-1461.

Galli, A.E.; C.F. LeCK \& R.T.T. Forman. 1976. Avian distribution patterns in forest islands of different sizes in central New jersey. The Auk, Washington, 93 (2) 356-364.

Gascon, C.; T.E. Lovejoy; R.O. Bierregaard JR.; J.R. Malcon; P.C.
Stouffer; H.L. Vasconcelos; W.L. Laurence; B. Zimmerman; M. ToCher \& S. Borges. 1999. Matriz habitat and species richness in tropical Forest remnants. Biological Conservation, Kidlington, 91 (2): 223-229.

Höfling, E. \& H.F. de A. Camargo. 1999. Aves no campus. São Paulo, EDUSP, $3^{\text {a }}$ ed., 163p.

IPARDES. 1993. Cobertura florestal e consumo de madeira, lenha e carvão nas micro regiões de Londrina, Maringá e Paranavaí: subsidio para uma política florestal no estado do Paraná. Curitiba, Instituto Paranaense de Desenvolvimento Econômico e Social, 44p.

KRÜGEL, M.M. \& L. dos Anjos. 2000. Bird communities in Forest remnants in the city of Maringá, Parana State, Southern Brazil. Ornitologia Neotropical, Montreal, 11 (2): 315-330.

Matarazzo-Neuberger, W.M. 1990. Lista das aves observadas na Cidade Universitária "Armando Salles de Oliveira", São Paulo, Brasil. Revista Brasileira de Biologia, São Carlos, 50 (2): 507-511.

Matarazzo-Neuberger, W.M. 1995. Comunidades de cinco parques e praças da Grande São Paulo, Estado de São Paulo. Ararajuba, Rio de Janeiro, 3 (1): 13-19.

Monteiro, M.P. \& D. Brandão. 1995. Estrutura da comunidade de aves do "Campus Samambaia" da Universidade Federal de Goiás, Goiânia, Brasil. Ararajuba, Rio de Janeiro, 3 (1) 21-26.

Murton, R.K.; E.H. Bucher; M. Nores, E. Gomes \& J. Reartes. 1974. The ecology of the Eared Dove (Zenaida auriculata) in Argentina. The Condor, Lawrance, 76 (1): 80-88u

Ranvaud, R.; K.C. De Freitas; E.H. Bucher; H.S. Dias; V.C.Avanjo \& C.C. AlberTs. 2001. Diet of Eared Doves (Zenaida auriculata, Aves, Columbidae) in a sugar-cane colony in South-eastern Brazil. Brazilian Journal of Biology, São Carlos, 61 (4): 651660.

Ridgely, R.S. \& G. Tudor. 1994. The birds of South America: The suboscines passerines. Oxford, Oxford University Press, vol. 2, XII+814p.

Scherer-Neto, P. \& F. Straube. 1995. Aves do Paraná: história, lista anotada e bibliografia. Curitiba, Edição dos autores, $79 \mathrm{p}$.

SICK, H. 1997. Ornitologia brasileira. Rio de Janeiro, Nova Fronteira, 912p.

SOARES, E.S. \& L. DOS ANJos. 1999. Efeito da fragmentação florestal sobre aves escaladoras de tronco e galho na região de Londrina, norte do Paraná, Brasil. Ornitologia Neotropical, Montreal, 10 (1) 61-68.

Stotz, D.F.; J.W. FitzPatrick; III T.A. PARKer \& D.K. Moskovits. 1996. Neotropical Birds: ecology and conservation. Chicago, University of Chicago Press, 410p.

Stouffer, P.C. \& R.O. Bierregaard Jr. 1995. Use of Amazonian forest fragments by understory insectivorous birds. Ecology, Washington, 76: 2429-2445.

Uezu, A.; J.P. Metzger \& J.M.E. Vielliard. 2005. Effects of structural and functional connectivity and patch size on 
the abundance of seven Atlantic Forest birds species. Biological Conservation, Kidlington, 123 (4): 507-519.

Warburton, N.H. 1997. Structure and conservation of forest avifauna in isolated rainforest remnants in tropical Australia, p. 190-206. In: W.F. Laurance \& R.O BierregaARd JR. (Eds). Tropical forest remnants: ecology, management, and consevation of fragmented communities. Chicago, The University of Chicago Press, XV+616p.
Westcott, P.W.; H. F. Mariño \& L. Dos Anjos. 2002. Observando aves em londrina: norte do Paraná - Brasil, Londrina, $\mathrm{XIV}+76 \mathrm{p}$.

WILLIS, E.O. 1979. The composition of avian communities in remanescent woodlots in southern Brazil. Papeis Avulsos de Zoologia, São Paulo, 33 (1): 1-25.

Willis, E.O. \& Y. Oniki. 2003. Aves do estado de São Paulo. Rio Claro, Edição dos Autores, 398p.

Recebido em 15.VI.2004; aceito em 18.I.2006.

Apêndice I. Lista geral das espécies de aves registradas na Universidade Estadual de Londrina durante a realização deste estudo. Mês, habitat e freqüência de ocorrência (FO, em porcentagem) das espécies nas amostragens. Taxonomia e nomenclatura seguem Comitê Brasileiro de Registros Ornitológicos (CBRO).

\begin{tabular}{|c|c|c|c|c|c|c|c|c|c|c|c|c|c|c|}
\hline \multirow{2}{*}{ Espécie } & \multicolumn{12}{|c|}{ Meses } & \multirow{2}{*}{ Habitat } & \multirow{2}{*}{ FO } \\
\hline & Jan & Fev & Mar & Abr & Mai & Jun & Jul & Ago & Set & Out & Nov & $\overline{\mathrm{Dez}}$ & & \\
\hline \multicolumn{15}{|l|}{ Tinamidae } \\
\hline Crypturellus parvirostris (Wagler, 1827) & & $x$ & & & $x$ & $x$ & $x$ & $x$ & $x$ & $x$ & $x$ & $x$ & $\mathrm{FL}, \mathrm{CP}, \mathrm{CA}$ & 31,2 \\
\hline Crypturellus tataupa (Temminck, 1815) & & $x$ & $x$ & & $x$ & $x$ & $x$ & $x$ & $x$ & $x$ & $x$ & $x$ & $\mathrm{FL}$ & 25,0 \\
\hline Nothura maculosa (Temminck, 1815) & $x$ & $x$ & & & $x$ & $x$ & $x$ & $x$ & $x$ & $x$ & $x$ & $x$ & $\mathrm{CA}$ & 47,6 \\
\hline \multicolumn{15}{|l|}{ Anatidae } \\
\hline \multicolumn{15}{|l|}{ Dendrocygninae } \\
\hline Dendrocygna viduata (Linnaeus, 1766) & & $x$ & & & & & & & $x$ & & $x$ & & AQ & 6,2 \\
\hline \multicolumn{15}{|l|}{ Anatinae } \\
\hline Amazonetta brasiliensis (Gmelin, 1789) & $x$ & $x$ & & & & $x$ & $x$ & $x$ & $x$ & $x$ & $x$ & $x$ & AQ & 35,4 \\
\hline \multicolumn{15}{|l|}{ Phalacrocoracidae } \\
\hline Phalacrocorax brasilianus (Gmelin, 1789)* & & & & & & & & & & & & & AQ & \\
\hline \multicolumn{15}{|l|}{ Ardeidae } \\
\hline Butorides striata (Linnaeus, 1758) & $x$ & $x$ & $x$ & & $x$ & & $x$ & $x$ & $x$ & & $x$ & $x$ & AQ & 25,0 \\
\hline Bubulcus ibis (Linnaeus, 1758) & & & & $x$ & $x$ & $x$ & $x$ & $x$ & $x$ & & $x$ & $x$ & $\mathrm{CA}$ & 31,2 \\
\hline Ardea alba Linnaeus, 1758 & & $x$ & & $x$ & & $x$ & $x$ & $x$ & $x$ & & $x$ & $x$ & AQ & 18,7 \\
\hline \multicolumn{15}{|l|}{ Cathartidae } \\
\hline Coragyps atratus (Bechstein, 1793) & $x$ & $x$ & & $x$ & $x$ & $x$ & $x$ & $x$ & $x$ & $x$ & $x$ & & $\mathrm{FL}, \mathrm{EA}, \mathrm{AA}, \mathrm{CA}$ & 29,2 \\
\hline \multicolumn{15}{|l|}{ Accipitridae } \\
\hline Elanoides forficatus (Linnaeus, 1758)*, ** & & & & & & & & & & & & & $\mathrm{CP}, \mathrm{CA}$ & \\
\hline Elanus leucurus (Vieillot, 1818) & & $x$ & $x$ & & $x$ & & & & & $x$ & & & $\mathrm{CP}, \mathrm{CA}$ & 8,3 \\
\hline Rupornis magnirostris (Gmelin, 1788) & $x$ & $x$ & $x$ & $x$ & $x$ & $x$ & $x$ & $x$ & $x$ & $x$ & $x$ & $x$ & $\mathrm{FL}, \mathrm{AA}, \mathrm{CP}$ & 75,0 \\
\hline Buteo brachyurus Vieillot, 1816 & & & & & & & & & & $x$ & & & AA & 4,2 \\
\hline \multicolumn{15}{|l|}{ Falconidae } \\
\hline Caracara plancus (Miller, 1777) & $x$ & & & $x$ & $x$ & $x$ & $x$ & & $x$ & $x$ & & $x$ & $\mathrm{FL}, \mathrm{EA}, \mathrm{AA}, \mathrm{CA}$ & 18,7 \\
\hline Milvago chimachima (Vieillot, 1816) & & & & & $x$ & $x$ & $x$ & $x$ & & & & & $\mathrm{CP}, \mathrm{CA}$ & 8,3 \\
\hline Falco sparverius Linnaeus, 1758 & & $x$ & $x$ & & $x$ & $x$ & $x$ & $x$ & & $x$ & $x$ & $x$ & $\mathrm{FL}, \mathrm{EA}, \mathrm{AA}$ & 29,2 \\
\hline Falco femoralis Temminck, 1822 & & $x$ & & & & & & & & & & & $\mathrm{CA}$ & 2,1 \\
\hline Falco peregrinus Tunstall, $1771^{*}$, ** & & & & & & & & & & & & & & \\
\hline
\end{tabular}


Apêndice I.Continuação.

Espécie

Rallidae

Aramides cajanea (Statius Muller, 1776)

Aramides saracura (Spix, 1825)

Laterallus melanophaius (Vieillot, 1819)

Gallinula chloropus (Linnaeus, 1758)

Jacanidae

Jacana jacana (Linnaeus, 1766)

Charadriidae

Vanellus chilensis (Molina, 1782)

Scolopacidae

Tringa solitaria Wilson, 1813**

Columbidae

Columbina talpacoti (Temminck, 1811)

Columbina squammata (Lesson, 1831)

Columbina picui (Temminck, 1813)

Columba livia Gmelin, 1789

Patagioenas picazuro (Temminck, 1813)

Patagioenas cayennensis (Bonnaterre, 1792)

Zenaida auriculata (Des Murs, 1847)

Leptotila verreauxi Bonaparte, 1855

Leptotila rufaxilla (Richard \& Bernard, 1792)

Geotrygon montana (Linnaeus, 1758)

Psittacidae

Aratinga leucophthalma (Statius Muller, 1776)

Aratinga auricapillus (Kuhl, 1820)*

Pyrrhura frontalis (Vieillot, 1817)*

Forpus xanthopterygius (Spix, 1824)

Pionopsitta pileata (Scopoli, 1769)

Pionus maximiliani (Kuhl, 1820)

Amazona aestiva (Linnaeus, 1758)*

Cuculidae

Cuculinae

Coccyzus melacoryphus Vieillot, 1817**

Piaya cayana (Linnaeus, 1766)

Crotophaginae

Crotophaga ani Linnaeus, 1758

Guira guira (Gmelin, 1788)

Neomorphinae

Tapera naevia (Linnaeus, 1766)

Strigidae

Megascops choliba (Vieillot, 1817)*

Athene cunicularia (Molina, 1782)

Nyctibidae

Nyctibius griseus (Gmelin, 1789)* $\frac{\text { Meses }}{\text { Jan Fev Mar Abr Mai Jun Jul Ago Set Out Nov Dez }}$

Habitat

FO

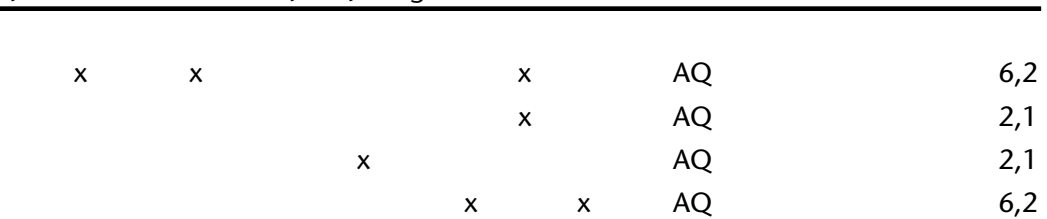

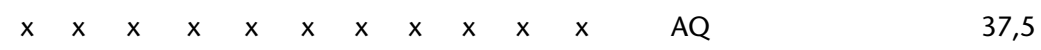

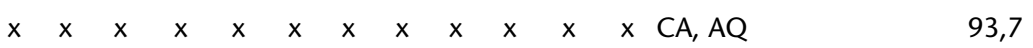

$\begin{array}{llll}x & x \quad 6 Q & 6,2\end{array}$

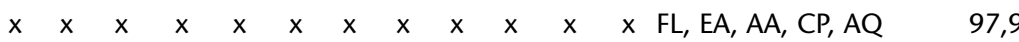

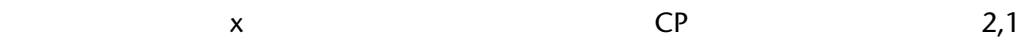

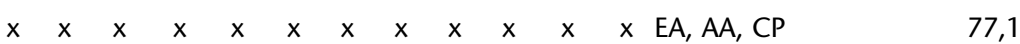

$\begin{array}{llllllllll}x & x & x & x & x & x & E A, C A & 20,8\end{array}$

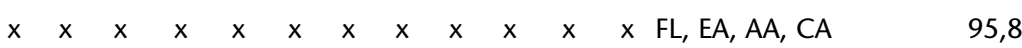

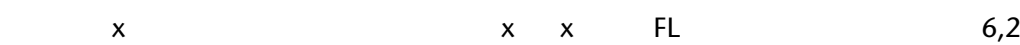

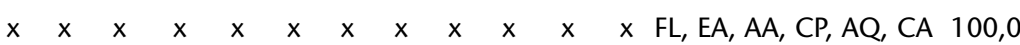

$\begin{array}{llllllllllllllllllllllll}x & x & x & x & x & x & x & x & x & x & x & x & F L & 75,0\end{array}$

$\begin{array}{lllllllllllllll}x & x & x & x & x & x & x & x & x & x & x & x & F L & 75,0 \\ x & x & & x & & x & x & x & x & & x & x & F L & 41,7\end{array}$

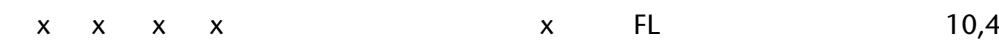

$\begin{array}{ll}\mathrm{FL}, \mathrm{AA} & 2,1\end{array}$

$\mathrm{FL}, \mathrm{AA}$

$\mathrm{FL}$

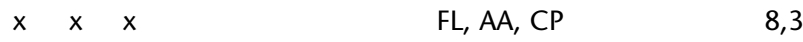

$\mathrm{FL}, \mathrm{AA} \quad 4,2$

$\mathrm{FL} \quad 2,1$

$\mathrm{FL}, \mathrm{AA}$

$\begin{array}{lllll}x & x & \mathrm{FL}, \mathrm{AA} & 6,2 \\ \mathrm{x} & \mathrm{x} & \mathrm{x} & \end{array}$

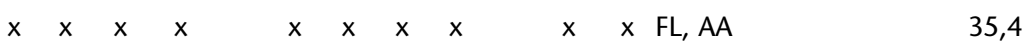

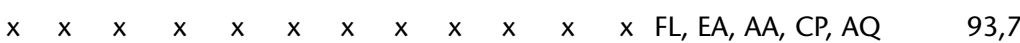

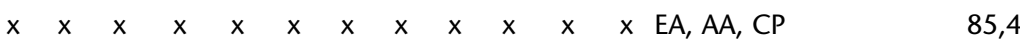

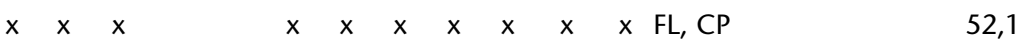

EA, AA

$x \quad x \quad x \quad x \quad x \quad x \quad x \quad x \quad x \quad x \quad x \quad x \quad C A$

79,2

$E A, A A$

Continua 
Apêndice I.Continuação.

\begin{tabular}{|c|c|c|c|c|c|c|c|c|c|c|c|c|c|c|}
\hline \multirow{2}{*}{ Espécie } & \multicolumn{12}{|c|}{ Meses } & \multirow{2}{*}{ Habitat } & \multirow{2}{*}{ FO } \\
\hline & \multicolumn{4}{|c|}{ Jan Fev Mar Abr } & \multicolumn{2}{|c|}{ Mai Jun } & \multicolumn{6}{|c|}{ Jul Ago Set Out Nov Dez } & & \\
\hline \multicolumn{15}{|l|}{ Caprimulgidae } \\
\hline Lurocalis semitorquatus (Gmelin, 1789)* & & & & & & & & & & & & & $\mathrm{EA}, \mathrm{AA}$ & \\
\hline Chordeiles minor (Forster, 1771)* & & & & & & & & & & & & & $\mathrm{EA}, \mathrm{AA}$ & \\
\hline Nyctidromus albicollis (Gmelin, 1789)* & & & & & & & & & & & & & $\mathrm{FL}, \mathrm{EA}, \mathrm{AA}$ & \\
\hline \multicolumn{15}{|l|}{ Apodidae } \\
\hline Chaetura meridionalis Hellmayr, 1907 & & $x$ & $x$ & $x$ & & & $x$ & $x$ & & $x$ & $x$ & $x$ & $\mathrm{FL}, \mathrm{EA}, \mathrm{AA}, \mathrm{CA}$ & 20,8 \\
\hline \multicolumn{15}{|l|}{ Trochilidae } \\
\hline \multicolumn{15}{|l|}{ Phaethornithinae } \\
\hline Phaethornis pretrei (Lesson \& Delattre, 1839) & & & & & & & $x$ & & $x$ & $x$ & & & $\mathrm{FL}, \mathrm{AA}$ & 6,2 \\
\hline Phaethornis eurynome (Lesson, 1832) & & & & & & & & & $x$ & & & & $\mathrm{FL}$ & 2,1 \\
\hline \multicolumn{15}{|l|}{ Trochilinae } \\
\hline Eupetomena macroura (Gmelin, 1788) & & $x$ & & & & & & & & $x$ & & $x$ & $E A, A A, C P$ & 10,4 \\
\hline Florisuga fusca (Vieillot, 1817)* & & & & & & & & & & & & & $E A, A A, C P$ & \\
\hline Anthracothorax nigricollis (Vieillot, 1817) & & $x$ & & & & & & & & $x$ & $x$ & $x$ & $E A, A A, C P$ & 10,4 \\
\hline $\begin{array}{l}\text { Chlorostilbon aureoventris (d'Orbigny \& } \\
\text { Lafresnaye, 1838) }\end{array}$ & $x$ & $x$ & $x$ & & & & $x$ & & & $x$ & $x$ & & $E A, A A, C P$ & 14,6 \\
\hline Thalurania glaucopis (Gmelin, 1788)* & & & & & & & & & & & & & $\mathrm{FL}, \mathrm{AA}$ & \\
\hline Hylocharis chrysura (Shaw, 1812) & & & & & & & & & & $x$ & & & $E A, A A, C P$ & 2,1 \\
\hline Leucochloris albicollis (Vieillot, 1818)* & & & & & & & & & & & & & $E A, A A, C P$ & \\
\hline Amazilia versicolor (Vieillot, 1818)* & & & & & & & & & & & & & $E A, A A, C P$ & \\
\hline Amazilia lactea (Lesson, 1832) & & & $x$ & & & & & & $x$ & $x$ & & & $E A, A A, C P$ & 10,4 \\
\hline Heliomaster squamosus (Temminck, 1823)* & & & & & & & & & & & & & $E A, A A, C P$ & \\
\hline \multicolumn{15}{|l|}{ Alcedinidae } \\
\hline Ceryle torquatus (Linnaeus, 1766) & $x$ & & $x$ & & $x$ & & $x$ & & $x$ & & $x$ & & $A Q$ & 35,4 \\
\hline Chloroceryle amazona (Latham, 1790) & & & & & & & $x$ & & $x$ & & $x$ & $x$ & $A Q$ & 12,5 \\
\hline Chloroceryle americana (Gmelin, 1788) & & $x$ & $x$ & & $x$ & $x$ & $x$ & & $x$ & $x$ & $x$ & $x$ & $A Q$ & 18,7 \\
\hline \multicolumn{15}{|l|}{ Ramphastidae } \\
\hline Selenidera maculirostris (Lichtenstein, 1823) & & & & & & $x$ & & & & & $x$ & & $\mathrm{FL}$ & 6,2 \\
\hline
\end{tabular}

Picidae

Picumnus temminckii Lafresnaye, 1845

Melanerpes candidus (Otto, 1796)

Melanerpes flavifrons (Vieillot, 1818)

Veniliornis spilogaster (Wagler, 1827)

Colaptes melanochloros (Gmelin, 1788)

Colaptes campestris (Vieillot, 1818)

Celeus flavescens (Gmelin, 1788)*

Dryocopus lineatus (Linnaeus, 1766)

Thamnophilidae

Hypoedaleus guttatus (Vieillot, 1816)

Mackenziaena severa (Lichtenstein, 1823)

Thamnophilus doliatus (Linnaeus, 1764)

Thamnophilus caerulescens Vieillot, 1816

$\begin{array}{llllllllll}x & x & x & x & x & x & & & & \end{array}$

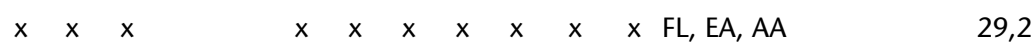

$\begin{array}{lllllllllllll}x & x & x & x & x & x & x & F L, E A, A A & 27,1\end{array}$

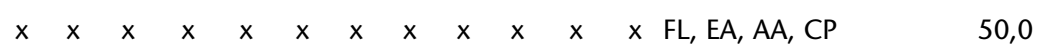

$\begin{array}{llllllllllllllllllllllllllllllll}x & x & x & x & x & x & x & x & F L, A A & 31,2\end{array}$

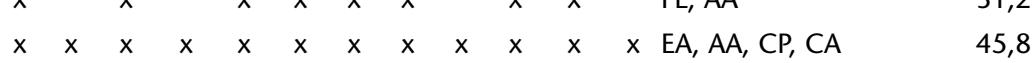

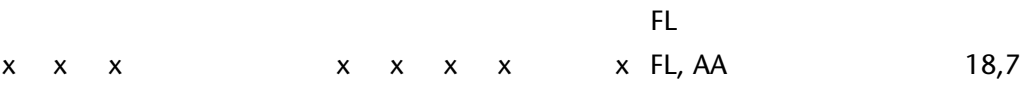

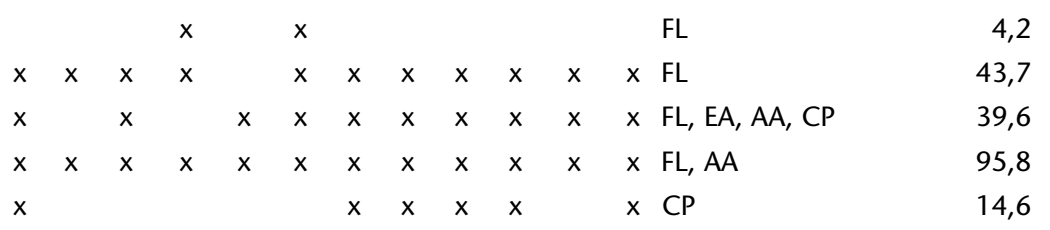

Thamnophilus ruficapillus Vieillot, 1816 
Apêndice I.Continuação.

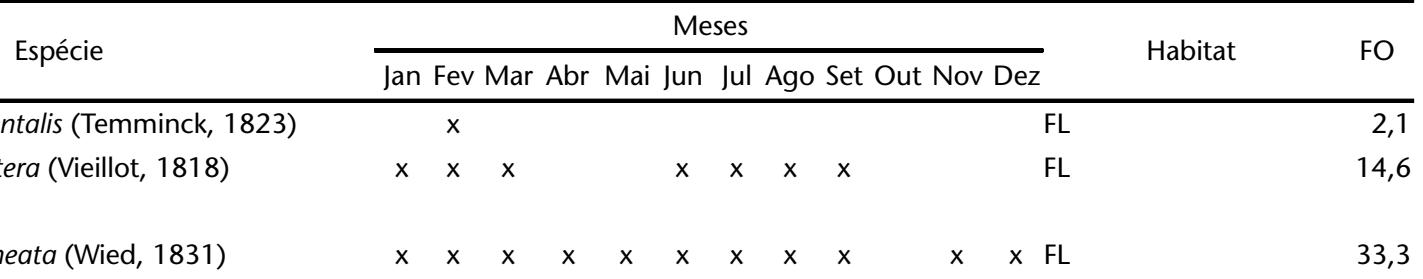

Dendrocolaptidae

Dendrocolaptes platyrostris Spix, 1825*

$\mathrm{FL}$

Furnariidae

Furnarius rufus (Gmelin, 1788)

Synallaxis ruficapilla Vieillot, 1819

Synallaxis frontalis Pelzeln, 1859

Synallaxis spixi Sclater, 1856

Xenops rutilans Temminck, 1821

Tyrannidae

Pipromorphinae

Leptopogon amaurocephalus Tschudi, 1846

Corythopis delalandi (Lesson, 1830)

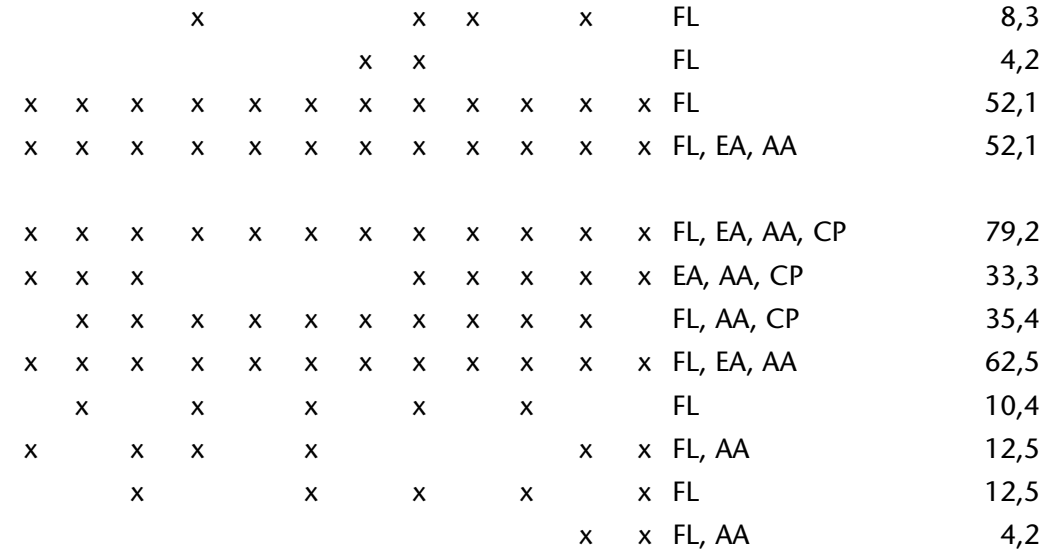

Poecilotriccus plumbeiceps (Lafresnaye, 1846)

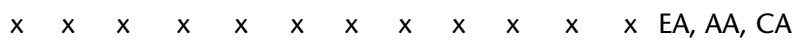

93,7

$\begin{array}{lllllllllllllllllllllllll}x & x & x & x & x & x & x & x & x & x & x & x & F L & 60,4\end{array}$

$\begin{array}{llllllllllllllllllllllllllllllllllllll}x & x & x & x & x & x & x & x & x & x & x & x & F L, C P & 54,2\end{array}$

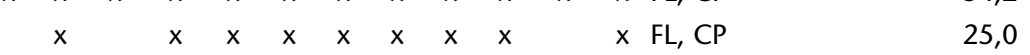

$\begin{array}{lllllll}x & x & x & x & F L, A A & 14,6\end{array}$

Todirostrum cinereum (Linnaeus, 1766)

Elaeniinae

Elaenia flavogaster (Thunberg, 1822)

Elaenia parvirostris Pelzeln, 1868**

Camptostoma obsoletum (Temminck, 1824)

Serpophaga subcristata (Vieillot, 1817)

Capsiempis flaveola (Lichtenstein, 1823)

Phylloscartes ventralis (Temminck, 1824)

Myiornis auricularis (Vieillot, 1818)

Tolmomyias sulphurescens (Spix, 1825)

Fluvicolinae

Myiophobus fasciatus (Statius Muller, 1776)

Lathrotriccus euleri (Cabanis, 1868)

Pyrocephalus rubinus (Boddaert, 1783)**

Satrapa icterophrys (Vieillot, 1818)

Colonia colonus (Vieillot, 1818)**

Machetornis rixosa (Vieillot, 1819)

Tyranninae

Legatus leucophaius (Vieillot, 1818)**

Myiozetetes similis (Spix, 1825)**

Pitangus sulphuratus (Linnaeus, 1766)

Myiodynastes maculatus (Statius Muller, 1776)**

Megarynchus pitangua (Linnaeus, 1766)**

Empidonomus varius (Vieillot, 1818)**

Griseotyrannus aurantioatrocristatus

(d'Orbigny \& Lafresnaye, 1837) $^{\star *}$

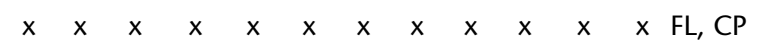

58,3

$\begin{array}{llllllllllllllll}x & x & x & x & x & x & x & x & x & x & F L & 50,0\end{array}$

$\begin{array}{llll}x & x & \mathrm{AA}, \mathrm{AQ} & 10,4\end{array}$

$x \quad x \quad F L \quad 4,2$

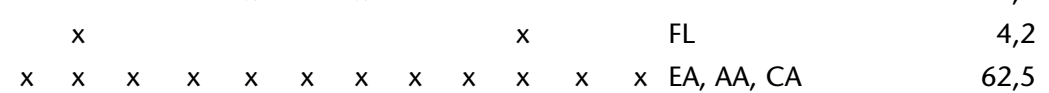

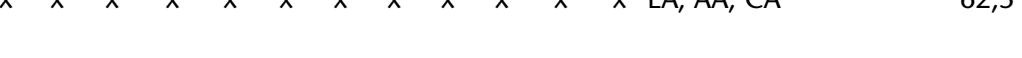

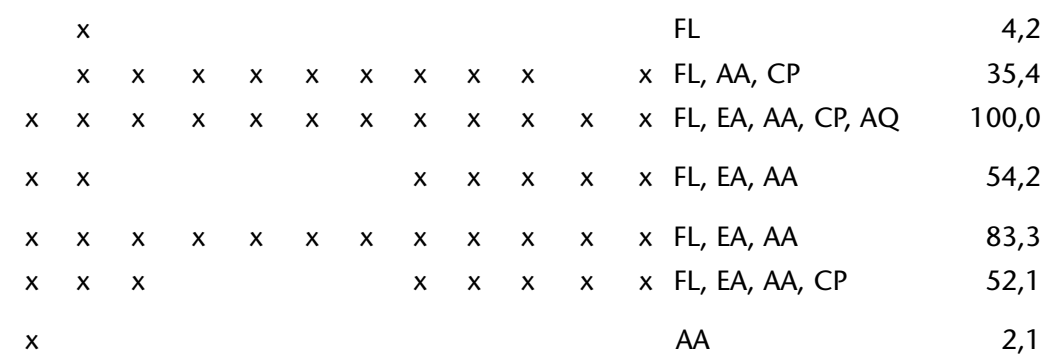

Continua

Revista Brasileira de Zoologia 23 (1): 145-156, março 2006 
Apêndice I.Continuação.

\begin{tabular}{|c|c|c|c|c|c|c|c|c|c|c|c|c|c|c|}
\hline \multirow{2}{*}{ Espécie } & \multicolumn{12}{|c|}{ Meses } & \multirow{2}{*}{ Habitat } & \multirow{2}{*}{ FO } \\
\hline & \multicolumn{4}{|c|}{ Jan Fev Mar Abr } & \multicolumn{2}{|c|}{ Mai Jun } & \multirow[t]{2}{*}{ Jul } & \multicolumn{5}{|c|}{ Ago Set Out Nov Dez } & & \\
\hline Tyrannus melancholicus Vieillot, 1819** & $\mathrm{x}$ & $x$ & $x$ & $x$ & & & & $\mathrm{x}$ & $x$ & $x$ & $x$ & $x$ & $\mathrm{FL}, \mathrm{EA}, \mathrm{AA}$ & 52,1 \\
\hline Tyrannus savana Vieillot, $1808^{* *}$ & $x$ & $\mathrm{x}$ & & & & & & $x$ & $x$ & $x$ & $x$ & $x$ & $\mathrm{FL}, \mathrm{EA}, \mathrm{AA}$ & 50,0 \\
\hline Sirystes sibilator (Vieillot, 1818)** & & $x$ & $x$ & & & & & & & & & & $\mathrm{FL}$ & 6,2 \\
\hline Myiarchus swainsoni Cabanis \& Heine, $1859^{* *}$ & & $x$ & $x$ & $x$ & & & & $x$ & $x$ & $x$ & & $x$ & $\mathrm{FL}, \mathrm{AA}$ & 25,0 \\
\hline \multicolumn{15}{|l|}{ Tityridae } \\
\hline Tityra inquisitor (Lichtenstein, 1823) & & & & $x$ & & & & & & & & $x$ & $\mathrm{FL}$ & 4,2 \\
\hline Tityra cayana (Linnaeus, 1766) & & & & & $x$ & & & & & & & & $\mathrm{FL}$ & 2,1 \\
\hline Pachyramphus polychopterus (Vieillot, 1818) & & & & & & & & & $x$ & & & $x$ & $\mathrm{FL}$ & 4,2 \\
\hline Pachyramphus validus (Lichtenstein, 1823)** & $x$ & $x$ & $x$ & & & & & $x$ & $x$ & $x$ & $x$ & $x$ & $\mathrm{FL}, \mathrm{AA}$ & 31,2 \\
\hline \multicolumn{15}{|l|}{ Vireonidae } \\
\hline Cyclarhis gujanensis (Gmelin, 1789) & $x$ & $x$ & $x$ & $x$ & $x$ & $x$ & $x$ & $x$ & $x$ & $x$ & $x$ & $x$ & $\mathrm{FL}, \mathrm{AA}$ & 81,2 \\
\hline Vireo olivaceus (Linnaeus, 1766$)^{* *}$ & & & & & & & & & $x$ & $x$ & & & $\mathrm{FL}, \mathrm{AA}$ & 6,2 \\
\hline \multicolumn{15}{|l|}{ Corvidae } \\
\hline Cyanocorax chrysops (Vieillot, 1818) & & & & & & & & & $x$ & & & & $\mathrm{FL}$ & 4,2 \\
\hline \multicolumn{15}{|l|}{ Hirundinidae } \\
\hline Tachycineta leucorrhoa (Vieillot, 1817) & & & & & & $x$ & $x$ & $x$ & & $x$ & $x$ & $x$ & $\mathrm{FL}, \mathrm{EA}, \mathrm{AA}, \mathrm{CP}, \mathrm{AQ}, \mathrm{CA}$ & 18,7 \\
\hline Progne tapera (Vieillot, 1817) & & & & & $x$ & $x$ & & & & & & & $\mathrm{FL}, \mathrm{EA}, \mathrm{AA}, \mathrm{CP}, \mathrm{AQ}, \mathrm{CA}$ & 6,2 \\
\hline Progne chalybea (Gmelin, 1789)** & & & $x$ & & $x$ & & & & & & $x$ & $x$ & $\mathrm{FL}, \mathrm{EA}, \mathrm{AA}, \mathrm{CP}, \mathrm{AQ}, \mathrm{CA}$ & 14,6 \\
\hline Pygochelidon cyanoleuca (Vieillot, 1817) & & $x$ & $x$ & $x$ & $x$ & $x$ & $x$ & $x$ & $x$ & $x$ & $x$ & $x$ & $\mathrm{FL}, \mathrm{EA}, \mathrm{AA}, \mathrm{CP}, \mathrm{AQ}, \mathrm{CA}$ & 52,1 \\
\hline Stelgidopteryx ruficollis (Vieillot, 1817) & & & & & $x$ & & $x$ & $x$ & $x$ & $x$ & & & $\mathrm{AA}, \mathrm{CP}, \mathrm{AQ}$ & 14,6 \\
\hline Hirundo rustica Linnaeus, $1758^{* *}$ & & & & & & & & & & & $x$ & & CA & 2,1 \\
\hline \multicolumn{15}{|l|}{ Troglodytidae } \\
\hline Troglodytes musculus Naumann, 1823 & $x$ & $x$ & $x$ & $x$ & $x$ & $x$ & $x$ & $x$ & $x$ & $x$ & $x$ & $x$ & $\mathrm{FL}, \mathrm{EA}, \mathrm{AA}, \mathrm{CP}$ & 100,0 \\
\hline Donacobius atricapilla (Linnaeus, 1766) & & $x$ & & & & & $x$ & $x$ & & & & $x$ & AQ & 12,5 \\
\hline \multicolumn{15}{|l|}{ Turdidae } \\
\hline Turdus rufiventris Vieillot, 1818 & $\mathrm{x}$ & $\mathrm{x}$ & $x$ & $x$ & $x$ & $\mathrm{x}$ & $x$ & $x$ & $x$ & $x$ & $x$ & $x$ & $\mathrm{FL}, \mathrm{AA}$ & 81,2 \\
\hline Turdus leucomelas Vieillot, 1818 & $x$ & $x$ & $x$ & $x$ & $x$ & $x$ & $x$ & $x$ & $x$ & $x$ & $x$ & $x$ & $\mathrm{FL}, \mathrm{EA}, \mathrm{AA}$ & 83,3 \\
\hline Turdus amaurochalinus Cabanis, 1850 & & $x$ & $x$ & $x$ & $x$ & $x$ & $x$ & $x$ & $x$ & $x$ & $x$ & $x$ & $\mathrm{FL}, \mathrm{EA}, \mathrm{AA}$ & 83,3 \\
\hline Turdus albicollis Vieillot, $1818^{* *}$ & & & & & & & & $x$ & & & & & $\mathrm{FL}$ & 2,1 \\
\hline \multicolumn{15}{|l|}{ Mimidae } \\
\hline Mimus saturninus (Lichtenstein, 1823) & $x$ & $x$ & $x$ & $x$ & & $x$ & $x$ & $x$ & $x$ & $x$ & $x$ & $x$ & $\mathrm{EA}, \mathrm{AA}, \mathrm{CP}$ & 45,8 \\
\hline \multicolumn{15}{|l|}{ Motacillidae } \\
\hline Anthus lutescens Pucheran, 1855 & & & & & $x$ & $x$ & $x$ & & & $x$ & & & $\mathrm{CA}$ & 18,7 \\
\hline \multicolumn{15}{|l|}{ Coerebidae } \\
\hline Coereba flaveola (Linnaeus, 1758) & $x$ & & $x$ & & $x$ & $x$ & $x$ & $x$ & $x$ & $x$ & $x$ & & $\mathrm{EA}, \mathrm{AA}, \mathrm{CP}$ & 27,1 \\
\hline \multicolumn{15}{|l|}{ Thraupidae } \\
\hline Cissopis leverianus (Gmelin, 1788) & & & $x$ & & & & & & & & & & $\mathrm{FL}$ & 2,1 \\
\hline Nemosia pileata (Boddaert, 1783) & & & & & & & $x$ & & & & & & $\mathrm{FL}, \mathrm{AA}$ & 2,1 \\
\hline Trichothraupis melanops (Vieillot, 1818) & $x$ & & & $x$ & $x$ & $x$ & & $x$ & & $x$ & & $x$ & $\mathrm{FL}$ & 14,6 \\
\hline Tachyphonus coronatus (Vieillot, 1822) & $x$ & $\mathrm{x}$ & $x$ & $x$ & $x$ & $x$ & $x$ & $x$ & $x$ & $x$ & $x$ & $x$ & $\mathrm{FL}$ & 72,9 \\
\hline Thraupis sayaca (Linnaeus, 1766) & $x$ & $x$ & $x$ & $x$ & $x$ & $x$ & $x$ & $x$ & $x$ & $x$ & $x$ & $x$ & $\mathrm{FL}, \mathrm{EA}, \mathrm{AA}, \mathrm{CP}$ & 91,7 \\
\hline Pipraeidea melanonota (Vieillot, 1819)* & & & & & & & & & & & & & $\mathrm{FL}, \mathrm{AA}$ & \\
\hline
\end{tabular}


Apêndice I.Continuação.

Espécie

Tersina viridis (Illiger, 1811)**

Dacnis cayana (Linnaeus, 1766)

Hemithraupis guira (Linnaeus, 1766)

Conirostrum speciosum (Temminck, 1824)

Emberizidae

Zonotrichia capensis (Statius Muller, 1776)

Ammodramus humeralis (Bosc, 1792)

Volatinia jacarina (Linnaeus, 1766)

Sporophila caerulescens (Vieillot, 1823)

Coryphospingus cucullatus (Statius Muller, 1776)

Cardinalidae

Saltator similis d'Orbigny \& Lafresnaye, 1837

Cyanocompsa brissonii (Lichtenstein, 1823)

Parulidae

Parula pitiayumi (Vieillot, 1817)

Geothlypis aequinoctialis (Gmelin, 1789)

Basileuterus culicivorus (Deppe, 1830)

Basileuterus leucoblepharus (Vieillot, 1817)

Icteridae

Cacicus haemorrhous (Linnaeus, 1766)

Gnorimopsar chopi (Vieillot, 1819)

Molothrus bonariensis (Gmelin, 1789)

Sturnella superciliaris (Bonaparte, 1850)

Fringillidae

Carduelis magellanica (Vieillot, 1805)* **

Euphonia chlorotica (Linnaeus, 1766)

Euphonia cyanocephala (Vieillot, 1818)**

Estrildidae

Estrilda astrild (Linnaeus, 1758)

Passeridae

Passer domesticus (Linnaeus, 1758)

$\frac{\text { Meses }}{\text { Jan Fev Mar Abr Mai Jun Jul Ago Set Out Nov Dez }}$

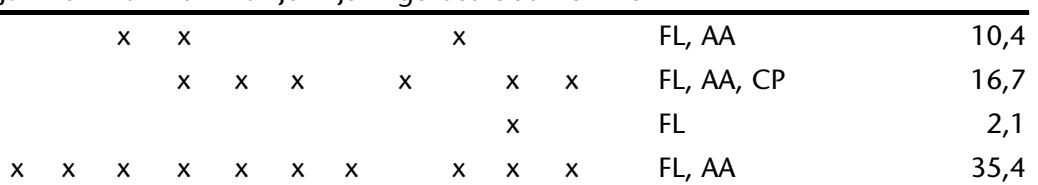

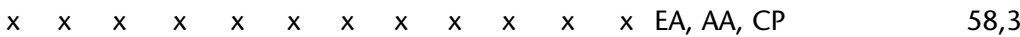

$\begin{array}{llllllllllllllllllllll}x & x & x & x & x & x & x & x & x & x & x & C P, C A & 27,1\end{array}$

$\begin{array}{llllllllllllllllllllllll}x & x & x & x & x & x & x & x & x & x & x & x & C P & 70,8\end{array}$

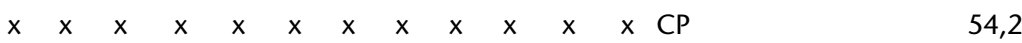

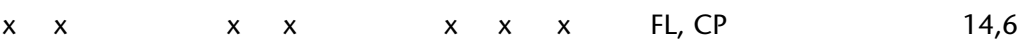

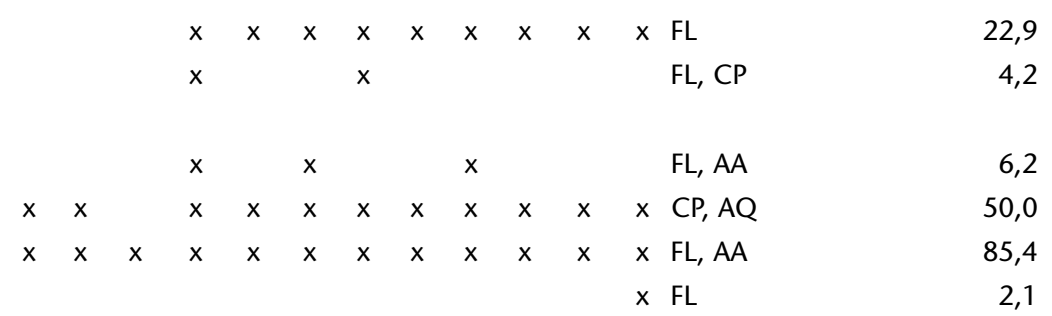

$\begin{array}{llll}x & \text { FL } & 4,2\end{array}$

AA

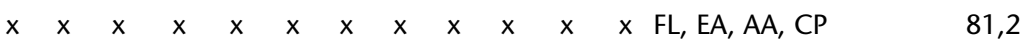

$\begin{array}{lllllllllllllllll}x & x & x & x & x & x & x & x & x & x & C A & 41,7\end{array}$

$\begin{array}{lllllllllllllllllll} & x & x & x & x & x & x & x & x & x & x & x & F L, E A, A A & 87,5\end{array}$

$x \times x \times x$ FL, AA 33,3

$\begin{array}{llll}x & \times & 8 & 8,3\end{array}$

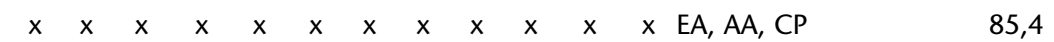

capoeira, (AQ) aquático, (CA) campo aberto. 